\title{
Predictors of Achieving Recommended Daily Physical Activity Among Anesthesiologists at a Large Tertiary Care Academic Center
}

\author{
Vesela P. Kovacheva ${ }^{\mathrm{a}, \mathrm{b}}$, Lawrence C. Tsen ${ }^{\mathrm{a}}$
}

\begin{abstract}
Background: The goal of the current study was to determine if the daily work patterns of anesthesiologists meet the recommended daily levels of activity.
\end{abstract}

Methods: Attending and resident anesthesiologists at a tertiary academic center were invited to participate. The subjects wore a pedometer during five regular clinical days at work and recorded the number of steps walked. The participants also completed the International Physical Activity Questionnaire (IPAQ) during one regular week. The results were analyzed using analysis of variance, Chi-square test and multivariate linear regression using STATA 12.1 .

Results: During work, attending, compared with senior and junior resident, anesthesiologists had the most steps $(5,953 \pm 1,213,5,153$ \pm 905 , and 5,710 $\pm 1,513$ steps, respectively, $\mathrm{P}=0.2)$. Outside work, senior residents had the highest level of activity (3,592 $\pm 1,626$ metabolic equivalent of task (MET)-minutes/week) compared to junior residents $(1,788 \pm 1,089 \mathrm{MET}$-minutes/week) and attending $(2,104$ $\pm 1,594 \mathrm{MET}$-minutes/week, $\mathrm{P}=0.005$ ); the percentage of recommended daily level of activity represented by this outside activity was senior residents $(78.5 \%)$, junior residents $(27 \%)$ and attending $(21 \%)$ anesthesiologists $(\mathrm{P}=0.002)$. When activity at and outside work was combined, most anesthesiologists met the recommended 10,000 steps daily, $\mathrm{P}<0.009$.

Conclusions: The daily physical activity of faculty and trainee anesthesiologists at work in a busy tertiary care is low active. However, when additional physical activity is pursued outside of work, most anesthesiologists met recommended daily levels of activity. These results highlight the inadequacy of daily activity at work, and the need to pursue additional physical activity outside of work; such awareness

Manuscript submitted September 25, 2017, accepted October 16, 2017

aDepartment of Anesthesiology, Perioperative and Pain Medicine, Brigham and Women's Hospital, Harvard Medical School, Boston, MA, USA

${ }^{b}$ Corresponding Author: Vesela P. Kovacheva, Department of Anesthesiology, Perioperative and Pain Medicine, Brigham and Women's Hospital, 75 Francis Street, Boston, MA 02115, USA. Email: vkovacheva@bwh.harvard.edu

doi: https://doi.org/10.14740/jocmr3208w can assist in promoting a healthy lifestyle.

Keywords: Occupational health; Anesthesiologist; Pedometer; Physical activity and health; Physical exercise; Physical activity on worksite

\section{Introduction}

The benefits of regular exercise on overall health are well known. Regular physical activity leads to primary and secondary prevention, and significant mortality reduction, from a number of chronic diseases, including obesity, coronary heart disease, heart failure, depression, dementia, cancer [1-4]. Conversely, physical inactivity is the fourth leading cause of death globally, accounting for 3.2 million deaths annually [5]. To promote and maintain health, most guidelines, including the World Health Organization's "Physical Activity for Health", recommend at least $150 \mathrm{~min}$ moderate exercise per week, which is considered equivalent to walking 10,000 steps daily [5-7]. Daily physical activity is frequently estimated through the use of surveys, such as the international physical activity questionnaire (IPAQ) $[8,9]$, or measured with pedometers $[10$, $11]$.

Physician well-being has been associated with patient safety. Lack of exercise, stress, and sleep deprivation result in impaired learning, poor work performance, and medical errors [12-14]. By contrast, physicians who exercise regularly have improved productivity and provide more efficient patient counseling and motivation [15-20].

Anecdotally, the perception of many anesthesiologists, particularly in large, busy academic centers such as ours, is that significant and sufficient amounts of physical activity occur within daily work patterns. Anesthesiologists, particularly attending anesthesiologists, perform and supervise a number of cases daily, often involving large cumulative distances and thus, physical activity. We designed the current study to determine and compare the total physical activity of attending and resident anesthesiologists at and outside work. We hypothesized that few anesthesiologists would achieve the recommended daily level of physical activity, as measured by number of steps, while at work, and that attending anesthesiologists 
Table 1. Subject Characteristics

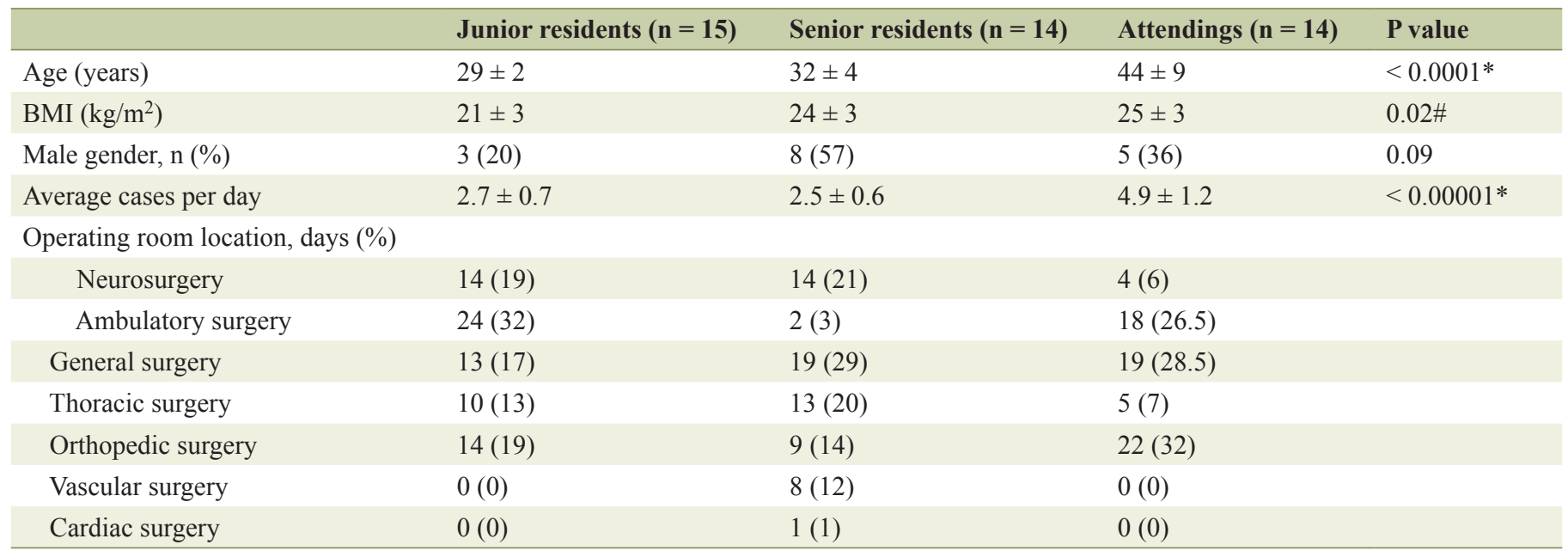

BMI: body mass index. All values are mean \pm standard deviation or number (percent). *Significance between attendings versus junior and senior residents. \#Significance between attendings versus junior residents.

would achieve a greater number of steps than senior and junior residents.

\section{Methods}

Following institutional review board/human research committee approval and registration with ClinicalTrials.gov (NCT01989494), all anesthesiology attendings and residents from the Department of Anesthesiology, Perioperative and Pain Medicine at the Brigham and Women's Hospital were invited to participate in the study through an announcement during the Department Grand Rounds and a single departmental email message. The inclusion criteria were: willingness to participate, full time clinical appointment and assignment in the Main Operating Room for five regular, non-call days. Subjects were excluded if unable to complete the study, or possessing limitations to physical activity or pregnant. A total of $45 \mathrm{sub}-$ jects were divided equally into three groups: attending, senior resident (postgraduate year (PGY) 3 and 4), and junior resident (PGY2) anesthesiologists.

Participants wore a triaxial pedometer (PedUSA Model PE771, Warminster, PA), and kept daily logs of the number of steps walked, the number of patients, and the operating room (OR) location. The pedometers were turned on and off upon arrival and departure from assigned lockers in the hospital. Before the collection of data, the accuracy of pedometer readings was verified for each participant through a manual step count over a 100-foot distance. The Brigham and Women's Hospital Main Operating Room Suite is organized on a pavilion, subspecialty principle with the Recovery Room and Pharmacy located equidistant from all OR locations included in this study. Participants also recalled their total physical activity outside of work over 7 days by completing the International Physical Activity Questionnaire (IPAQ), short version (www.ipaq. ki.se). Total physical activity outside work was calculated in steps per day, using a standardized, accepted conversion of 60 min of brisk walking resulting in 6,000 steps or 198 metabolic equivalent of task (MET)-minutes [7].

Continuous variables are presented as mean \pm standard deviation (SD) and categorical variables are presented as number, percent. The comparisons between the groups were performed using the analysis of variance (ANOVA) test followed by Bonferroni correction for continuous variables or Chi-square and Fisher exact test for categorical variables. Multivariate linear regression was used to study the relationship between continuous variables and to account for possible confounding factors such as age, body mass index (BMI) and number of cases for the day. The statistical software STATA 12.1 was used with significance reported if $\mathrm{P}<0.05$.

\section{Results}

From a total of 45 participants, data were excluded from one attending and one senior resident who dropped out of the study; three subjects had some missing data but were kept in the study. Participant characteristics and the OR assignment are provided (Table 1). Attending anesthesiologists were significantly older than residents $(\mathrm{P}<0.0001)$, and had a higher BMI than the junior residents $(P=0.02)$. There were no differences in gender composition among the groups $(\mathrm{P}=0.09)$. The attending anesthesiologists had more cases $(4.9 \pm 1.2)$ per day, compared to senior $(2.5 \pm 0.6)$ and junior residents $(2.7$ $\pm 0.7)(\mathrm{P}<0.00001)$. Attending anesthesiologists did most of their work in orthopedic, general and ambulatory surgery, senior residents in general, thoracic and neurosurgery, and junior residents in ambulatory, orthopedic and neurosurgery.

During work, the average number of steps decreased from attending to junior and senior resident anesthesiologists $(5,953$ $\pm 1,213,5,710 \pm 1,513$, and $5,153 \pm 905, \mathrm{P}=0.2$ ), respectively (Table 2). Only two anesthesiologists on three different days fulfilled the 10,000 steps daily requirement at work. There were no correlations between the average number of steps and 
Table 2. Results

\begin{tabular}{|c|c|c|c|c|}
\hline & Junior residents $(\mathrm{n}=15)$ & Senior residents $(n=14)$ & Attendings $(n=14)$ & P value \\
\hline \multicolumn{5}{|l|}{ At work } \\
\hline Average steps per day & $5,710 \pm 1,513$ & $5,153 \pm 905$ & $5,953 \pm 1,213$ & 0.2 \\
\hline \multicolumn{5}{|l|}{ Outside of work } \\
\hline Vigorous PA, MET-minutes per week & $779 \pm 751$ & $1,422 \pm 1,182$ & $969 \pm 1395$ & 0.3 \\
\hline Total PA, MET-minutes per week & $1,788 \pm 1,089$ & $3,592 \pm 1,626$ & $2,104 \pm 1,594$ & $0.005 \S$ \\
\hline Level 3, HEPA active, n (\%) & $4(27)$ & $11(78.5)$ & $3(21)$ & $0.002 \S$ \\
\hline Converted PA, steps per day & $7,739 \pm 4,712$ & $15,548 \pm 7,038$ & $9,110 \pm 6,900$ & $0.005 \S$ \\
\hline \multicolumn{5}{|l|}{ Total } \\
\hline
\end{tabular}

PA: physical activity; MET: metabolic equivalent of task; HEPA: health-enhancing physical activity. All values are mean \pm standard deviation or number (percent). *Significance between attendings versus junior and senior residents. §Significance between senior residents versus attendings and junior residents.

cases per day, or the amount of physical activity at and outside of work, even after adjusting for the number of cases per day.

Outside of work, the senior residents did more moderate exercise $(875 \pm 658 \mathrm{MET}$-minutes/week) compared to the junior residents $(272 \pm 309$ MET-minutes/week $)$ and attendings (292 \pm 461 MET-minutes/week), $\mathrm{P}=0.004$ (Table 2). Similarly, the total physical activity was higher in senior residents $(3,592 \pm 1,626$ MET-minutes/week) compared to junior residents $(1,788 \pm 1,089 \mathrm{MET}-$ minutes/week) and attendings $(2,104 \pm 1,594$ MET-minutes/week $)(\mathrm{P}=0.005)$. Based on IPAQ analysis, only $27 \%$ of junior resident and $21 \%$ of attendings met the requirement for health-enhancing physical activity, while $78.5 \%$ of the senior residents satisfied that level of activity $(\mathrm{P}=0.002)$. When converted to daily steps, the average outside of work physical activity was equivalent to 7,739 \pm 4,712 steps per day for junior resident; $15,548 \pm 7,038$ steps per day for senior resident and 9,110 $\pm 6,900$ steps per day for attending anesthesiologists $(\mathrm{P}=0.005)$.

When combining the exercise at work and outside work, most anesthesiologists met the recommended daily 10,000 steps, $\mathrm{P}<0.009$.

\section{Discussion}

In our study, we observed that anesthesiologists of all levels who were assigned to work in the OR of a large, tertiary care medical center did not achieve the WHO recommended daily activity level at work; no significant differences were observed in the number of steps among attending, senior and junior residents, and no association was found to age, gender, BMI or workload. When physical activity within and outside of work was combined, most anesthesiologists achieved the recommended daily activity level; overall, senior residents had the highest total activity level.

Approximately 50\% of recommended daily activity levels were achieved by work related physical activity for all anesthesiologist levels. These findings are consistent with other reports of occupational physical activity among anesthesiologists, although global differences appear to exist. Whereas tertiary care cardiac attending and fellow anesthesiologists in the United States walked an average of 5,553 $\pm 1,762$ daily steps [21], consultant anesthesiologists in Australia and consultant and trainee anesthesiologists in the United Kingdom walked an average of 4,770 (range 1,667 - 9,630) [22] and 3,694 (range 1,444 - 7,712) [23], respectively. By contrast, in Japan, the physical activity of anesthesiology supervisors, instructors, and residents was observed to be 11,599, 8,399, and 7,249, respectively [24].

The distinct difference between attending and resident anesthesiologists in Japan [24, 25], which contrasts to our findings and the reports cited from Australia and the United Kingdom [23] likely reflects variations in trainee supervision. In our practice, one attending anesthesiologist supervises two ORs, each with a resident anesthesiologist; by contrast, in Japan, two attending anesthesiologists supervise 12 ORs with a total of 10 - 12 residents. Differences in expectations for intraoperative teaching may also exist and alter physical activity; our attending-trainee dyads spend significant amounts of time during each case to discuss relevant topics.

Outside of work, the majority of senior resident anesthesiologists achieved a higher amount of exercise than attending and junior resident anesthesiologists. Although the reasons for these findings were not examined in our study, physicians have observed differences in the level of engagement with exercise, due to time prioritization, attitude towards exercise and family commitments. A study of consultant anesthesiologists in Australia found that the main reasons not to engage in regular exercise included being too busy $(70 \%)$, family commitments $(67 \%)$, fatigue (40\%) and lack of interest (18\%) [22]. In internal medicine, a large survey of attendings, fellows, residents, and medical students in the United States, found attendings 
and medical students more likely to meet established physical activity guidelines [26]. Internal medicine attendings in other studies have reported higher levels of exercise than residents [27], with only $41 \%$ of medicine residents meeting the requirements for adequate physical exercise [28]; interestingly, residents expressed more negative attitudes regarding exercise, with attendings being more willing to engage in exercise programs. In Canada, medical residents had lower physical activity levels than medical students [29].

Our study has several limitations. First, the participation in our study was introduced to our department at a Grand Rounds presentation and by email and was completely voluntary, which may have attracted the most active participants. Additional limitation is the small number of study participants. These potential biases may have been mitigated by our sample size representing approximately $50 \%$ of our resident component. Second, although we measured number of steps at work as a surrogate for physical activity, anesthesiologists do a variety of manual activities in their daily work patterns, including moving stretchers and equipment, lifting patients, and applying and removing monitors. An alternative approach could have been tracking energy expenditure [25], but these measurements have been shown to be unreliable between different brands of accelerometers [11,30]. Third, although it would have been potentially valuable to specifically evaluate and contrast the daily physical activity of the attending-trainee dyads assigned to work together, we opted against this given the potential conflict this may have presented to those responsible for making the clinical schedule. Fourth, it is possible that our study subjects intentionally performed more steps due to the availability of the pedometer and participation in our study. To minimize this effect, we instructed participants to be aware of this bias and that the intent was not to "beat" their daily activity average each day; better mechanisms would have been to blind participants to the results of their activity meters and have more expensive devices that would allow remote recording of the daily totals; however, we required that our participants record and reset their pedometers each day. Fifth, physical activity after work hours was quantified using the IPAQ, which does not have a tight correlation with objective measures for exercise [31]. We decided to proceed with this method, due to its wide use [22, 23, 26, 27] and ability to record a variety of activities like swimming and cycling, which cannot be quantified with pedometers. Sixth, both parts of the study depended on subjects robustly and honestly recording their pedometer results and IPAQ questionnaire, which has the potential to introduce validity bias. In addition, it is possible that some of the differences between the observed groups in our study result from the different OR location, which also may limit the external validity of our study. To minimize this bias, we excluded physicians working at remote locations like endoscopy and labor floor.

Many reasons have been cited for anesthesiologists, as well as other physicians, not to engage in a regular exercise programs, including family commitments, lack of time, and attitudes towards exercise and fatigue [22, 27]. Residency is considered a stressful time for physicians and there are numerous guidelines about promotion of healthy lifestyle with restriction of work hours and sufficient time to rest. However, there is insufficient objective data whether other measures of healthy lifestyle, like physical activity, are being adequately promoted or achieved. We find that the beginning of residency is associated with the highest period of physical inactivity. Studies from other industries have shown that adequate physical activity can improve psychological and overall health and decrease the number of days absent from work $[32,33]$. Residents tend to express higher stress levels when there is a lack of exercise [27] and as a consequence, further studies regarding barriers to exercise, especially early in residency, are needed. Possible solutions from other industries include workplace health promotion programs $[34,35]$, incorporating short physical activity breaks during the day [36], and addressing motivational barriers [37-39].

\section{Conclusion}

The success and perhaps longevity of an individual's career can be influenced by health habits including physical activity, but this connection is infrequently promoted in training and physician wellness programs [40]. The daily physical activity of faculty and trainee anesthesiologists at work in a busy tertiary care is low active. However, when additional physical activity is pursued outside of work, most anesthesiologists met recommended daily levels of activity. These results highlight the need to pursue additional physical activity outside of work, and can assist in promoting awareness of a healthy lifestyle.

\section{Acknowledgments}

The authors would like to thank Mr. C. Huang for help with statistics and Dr. A. Banooni for help with subject recruitment.

\section{Conflict of Interest}

The authors declare no conflict of interest.

\section{Financial Disclosures}

None.

\section{Author Contributions}

All authors listed in the manuscript have contributed to the plan of the study, analysis of the data, draft of the manuscript or critical revisions and have approved the final version of the manuscript.

\section{References}

1. Pedersen BK, Saltin B. Exercise as medicine - evidence 
for prescribing exercise as therapy in 26 different chronic diseases. Scand J Med Sci Sports. 2015;25(Suppl 3):1-72.

2. Chang P, Nead KT, Olin JW, Myers J, Cooke JP, Leeper NJ. Effect of physical activity assessment on prognostication for peripheral artery disease and mortality. Mayo Clin Proc. 2015;90(3):339-345.

3. Myers J, Nead KT, Chang P, Abella J, Kokkinos P, Leeper NJ. Improved reclassification of mortality risk by assessment of physical activity in patients referred for exercise testing. Am J Med. 2015;128(4):396-402.

4. Lollgen H, Bockenhoff A, Knapp G. Physical activity and all-cause mortality: an updated meta-analysis with different intensity categories. Int J Sports Med. 2009;30(3):213224.

5. WHO. Global recommendations on physical activity for health. http://wwwwhoint/dietphysicalactivity/publications/9789241599979/en/. 2010.

6. Garber CE, Blissmer B, Deschenes MR, Franklin BA, Lamonte MJ, Lee IM, Nieman DC, et al. American College of Sports Medicine position stand. Quantity and quality of exercise for developing and maintaining cardiorespiratory, musculoskeletal, and neuromotor fitness in apparently healthy adults: guidance for prescribing exercise. Med Sci Sports Exerc. 2011;43(7):1334-1359.

7. Tudor-Locke C, Craig CL, Brown WJ, Clemes SA, De Cocker K, Giles-Corti B, et al. How many steps/day are enough? For adults. Int J Behav Nutr Phys Act. 2011;8:79.

8. Kim Y, Park I, Kang M. Convergent validity of the international physical activity questionnaire (IPAQ): metaanalysis. Public Health Nutr. 2013;16(3):440-452.

9. Craig CL, Marshall AL, Sjostrom M, Bauman AE, Booth ML, Ainsworth BE, Pratt M, et al. International physical activity questionnaire: 12 -country reliability and validity. Med Sci Sports Exerc. 2003;35(8):1381-1395.

10. Tudor-Locke C, Williams JE, Reis JP, Pluto D. Utility of pedometers for assessing physical activity: convergent validity. Sports Med. 2002;32(12):795-808.

11. Ferguson T, Rowlands AV, Olds T, Maher C. The validity of consumer-level, activity monitors in healthy adults worn in free-living conditions: a cross-sectional study. Int J Behav Nutr Phys Act. 2015;12:42.

12. Baldwin PJ, Dodd M, Wrate RW. Young doctors' health I. How do working conditions affect attitudes, health and performance? Soc Sci Med. 1997;45(1):35-40.

13. Buddeberg-Fischer B, Stamm M, Buddeberg C, Bauer G, Hammig O, Klaghofer R. [Work stress, health and satisfaction of life in young doctors. Results of a longitudinal study in Switzerland]. Dtsch Med Wochenschr. 2008;133(47):2441-2447.

14. Kang SH, Boo YJ, Lee JS, Han HJ, Jung CW, Kim CS. High occupational stress and low career satisfaction of Korean surgeons. J Korean Med Sci. 2015;30(2):133139.

15. Frank E, Bhat Schelbert K, Elon L. Exercise counseling and personal exercise habits of US women physicians. J Am Med Womens Assoc (1972). 2003;58(3):178-184.

16. Howe M, Leidel A, Krishnan SM, Weber A, Rubenfire M, Jackson EA. Patient-related diet and exercise counseling: do providers' own lifestyle habits matter? Prev Cardiol.
2010;13(4):180-185.

17. Frank E, Tong E, Lobelo F, Carrera J, Duperly J. Physical activity levels and counseling practices of U.S. medical students. Med Sci Sports Exerc. 2008;40(3):413-421.

18. Abramson S, Stein J, Schaufele M, Frates E, Rogan S. Personal exercise habits and counseling practices of primary care physicians: a national survey. Clin J Sport Med. 2000;10(1):40-48.

19. Morishita Y, Numata A, Miki A, Okada M, Ishibashi K, Takemoto F, Ando Y, et al. Primary care physicians' own exercise habits influence exercise counseling for patients with chronic kidney disease: a cross-sectional study. BMC Nephrol. 2014;15:48.

20. Rogers LQ, Gutin B, Humphries MC, Lemmon CR, Waller JL, Baranowski T, Saunders R. A physician fitness program: enhancing the physician as an "exercise" role model for patients. Teach Learn Med. 2005;17(1):27-35.

21. Abd TT, Kobylivker A, Perry A, Miller Iii J, Sperling L. Work-related physical activity among cardiovascular specialists. Clin Cardiol. 2012;35(2):78-82.

22. Soh M, Deam RK, Kluger R. 10,000 reasons to step out exercise patterns and pedometer evaluation of consultant anaesthetists. Anaesth Intensive Care. 2006;34(3):347352.

23. Cuthill JA, Fitzpatrick K, Glen J. Anaesthesia - a sedentary specialty? Accelerometer assessment of the activity level of anaesthetists while at work. Anaesthesia. 2008;63(3):279-283.

24. Mizuno J, Kato S, Morita S. [Energy expenditure of anesthesiologists estimated from number of steps]. Masui. 2009;58(12):1549-1553.

25. Tanaka H, Kawamata T, Gen-No H, Nose H, Kawamata M. Evaluation of the physical activity of anesthesiologists in the operating room during daily work using a triaxial accelerometer. Arch Environ Occup Health. 2015;70(2):77-80.

26. Stanford FC, Durkin MW, Blair SN, Powell CK, Poston MB, Stallworth JR. Determining levels of physical activity in attending physicians, resident and fellow physicians and medical students in the USA. Br J Sports Med. 2012;46(5):360-364.

27. Williams AS, Williams CD, Cronk NJ, Kruse RL, Ringdahl EN, Koopman RJ. Understanding the exercise habits of residents and attending physicians: a mixed methodology study. Fam Med. 2015;47(2):118-123.

28. Rogers LQ, Gutin B, Humphries MC, Lemmon CR, Waller JL, Baranowski T, Saunders R. Evaluation of internal medicine residents as exercise role models and associations with self-reported counseling behavior, confidence, and perceived success. Teach Learn Med. 2006;18(3):215-221.

29. Rye PL, Reeson ME, Pekrul CM, Asfour NA, Kundapur R, Wilson MP, Pausjenssen AM, et al. Comparing health behaviours of internal medicine residents and medical students: an observational study. Clin Invest Med. 2012;35(1):E40-44.

30. Kumahara H, Ayabe M, Ichibakase M, Tashima A, Chiwata M, Takashi T. Validity of activity monitors worn at multiple nontraditional locations under controlled and 
free-living conditions in young adult women. Appl Physiol Nutr Metab. 2015;40(5):448-456.

31. Lee PH, Macfarlane DJ, Lam TH, Stewart SM. Validity of the International Physical Activity Questionnaire Short Form (IPAQ-SF): a systematic review. Int J Behav Nutr Phys Act. 2011;8:115.

32. Bernaards CM, Jans MP, van den Heuvel SG, Hendriksen IJ, Houtman IL, Bongers PM. Can strenuous leisure time physical activity prevent psychological complaints in a working population? Occup Environ Med. 2006;63(1):1016.

33. Leino-Arjas P, Solovieva S, Riihimaki H, Kirjonen J, Telama R. Leisure time physical activity and strenuousness of work as predictors of physical functioning: a 28 year follow up of a cohort of industrial employees. Occup Environ Med. 2004;61(12):1032-1038.

34. Pereira MJ, Coombes BK, Comans TA, Johnston V. The impact of onsite workplace health-enhancing physical activity interventions on worker productivity: a systematic review. Occup Environ Med. 2015;72(6):401-412.

35. Robroek SJ, Lindeboom DE, Burdorf A. Initial and sustained participation in an internet-delivered long-term worksite health promotion program on physical activity and nutrition. J Med Internet Res. 2012;14(2):e43.

36. Babey $\mathrm{SH}, \mathrm{Wu} \mathrm{S}$, Cohen $\mathrm{D}$. How can schools help youth increase physical activity? An economic analysis comparing school-based programs. Prev Med. 2014;69(Suppl 1):S55-60.

37. Zarrett N, Sorensen C, Cook BS. Physical and social-motivational contextual correlates of youth physical activity in underresourced afterschool programs. Health Educ Behav. 2015;42(4):518-529.

38. Deliens T, Deforche B, De Bourdeaudhuij I, Clarys P. Determinants of physical activity and sedentary behaviour in university students: a qualitative study using focus group discussions. BMC Public Health. 2015;15:201.

39. Mailey EL, McAuley E. Impact of a brief intervention on physical activity and social cognitive determinants among working mothers: a randomized trial. J Behav Med. 2014;37(2):343-355.

40. Harms BA, Heise CP, Gould JC, Starling JR. A 25-year single institution analysis of health, practice, and fate of general surgeons. Ann Surg. 2005;242(4):520-526; discussion 526-529. 